\title{
Application of the Deming PDSA Cycle as a Patient Experience Management Tool to Improve HIV Care. An Action Research in Under- Resourced Settings of Jinja-Uganda
}

\author{
Balidawa John \\ Lecturer, UNICAF University, Uganda
}

\begin{abstract}
The study to determine the effect of patient experience management using the Deming PDSA cycle on the quality of HIV care was conducted in under-resourced settings of Uganda. The interventional study had 437 files studied, and 224 adult HIV clients with poor HIV care outcomes of; missed appointments for more than two weeks, poor adherence to treatment of $<85 \%$, and unsuppressed viral load of > 1000 viral copies per milliliter were interviewed at baseline and end line. The study revealed a $13.6 \%$ prevalence of the study HIV care outcomes among all the client files, the majority of which were for patients in care for more than four years. $11.8 \%, 65.2 \%$, and $46.5 \%$ were due to poor adherence to treatment, two weeks missed appointments, and unsuppressed viral load at baseline, which reduced to $4.7 \%, 25.1 \%$, and $31.5 \%$ at end-line assessment, respectively. $80 \%$ of the studied clients were retention in care between the baseline and end-line assessments. $7.7 \%$ of the study files had never had a viral load done to the clients at baseline, which reduced to $1.4 \%$ at the end line. $34.5 \%$ of the study client files were overdue for a viral load at baseline but only $16.8 \%$ at end-line assessment. The study also showed a general improvement on the patient experience before and after interventions. In conclusion, Patient Experience Management using the Deming PDSA cycles improves the quality of HIV care in under-resourced settings.
\end{abstract}

Keywords: Deming PDSA cycles, Management, Patient Experience.

\section{Introduction}

Quality healthcare is achieved through continuous quality improvement, which concept was started in 1982 by Deming, who demonstrated the science of improvement using the Plan-Do-Study-Act (PDSA) cycle [1]. Patient-centred care, one of the dimensions of quality healthcare, is well documented in the Uganda National health sector quality improvement framework and strategic plan 2015/16-2019/20 as one of the approaches to improve the quality of care in the Ugandan healthcare system. The study of "Application of the Deming PDSA cycle as a patient experience management tool to improve HIV care, was an action research in under resourced settings of Jinja- Uganda" to provides an alternative to the current quality improvement approaches. Patient experience is a patient-centred care, a concept that started in the 1980s, create a positive patient experience, and it is until today relevant in the healthcare environment [2].

In recent years, perceptions of performance and quality of healthcare organizations have begun to move beyond examining the provision of excellent clinical care alone, but to consider and embrace the patient experience as an important indicator [3]. Patient experience is defined as "the sum of all interactions, shaped by an organization's culture, that influence patient perceptions, across the continuum of care" [2] [3]. It is an important factor in patient perception of quality, leading to better utilization and adherence to treatment [4]. It is known that poor 
adherence to treatment in HIV care leads to unsuppressed viral load, high chances of HIV transmission, and drug resistance hence the use of second and third-line regimens that are expensive.

\section{Background}

The quality of HIV care and management in Uganda is unsatisfactory, with $59.6 \%$ prevalence of viral suppression nationally and $48.8 \%$ for the East central region where Jinja is located. Jinja district, with 28486 active HIV patients in care, has 2848 patients on second-line regimen of ARVs. The district also has $7.9 \%$ (2250) miss appointments, $5.3 \%$ (1509) poor adherence to treatment, and $10.4 \%$ (2962) of HIV clients with unsuppressed viral load. The poor HIV care outcomes are attributed to poor patient adherence to treatment. The situation was worsened by the adoption of the Test and Treat Guidelines of WHO in 2016 by Uganda, which increased the number of HIV clients into care from 21571 to 28486, amidst limited resources, ARV stock-outs, and lack of service providers skilled in adherence counseling.
To determine the prevalence of poor HIV care outcomes among patients in care, to determine the patient experience of clients with poor HIV care outcomes, and to determine the effect of application of Deming PDSA cycle as a patient experience management tool on the quality of HIV care.

\section{Justification}

The study was intended to provide new ideas on using patient experience management as an approach to improve the quality of HIV care in under-resourced settings. The study was to support the decision-makers and other stakeholders to make decisions, policies, and guidance based on evidence. The study was also to improve on patient-centred care for improved patient adherence to care and treatment reduced missed appointments and improved viral suppression and health status of HIV patients. It was anticipated that the public health outcome of the study will contribute to reduced HIV transmission with improved viral suppression. The policy makers are to make policies with evidence from the study.

\section{Study Objectives}

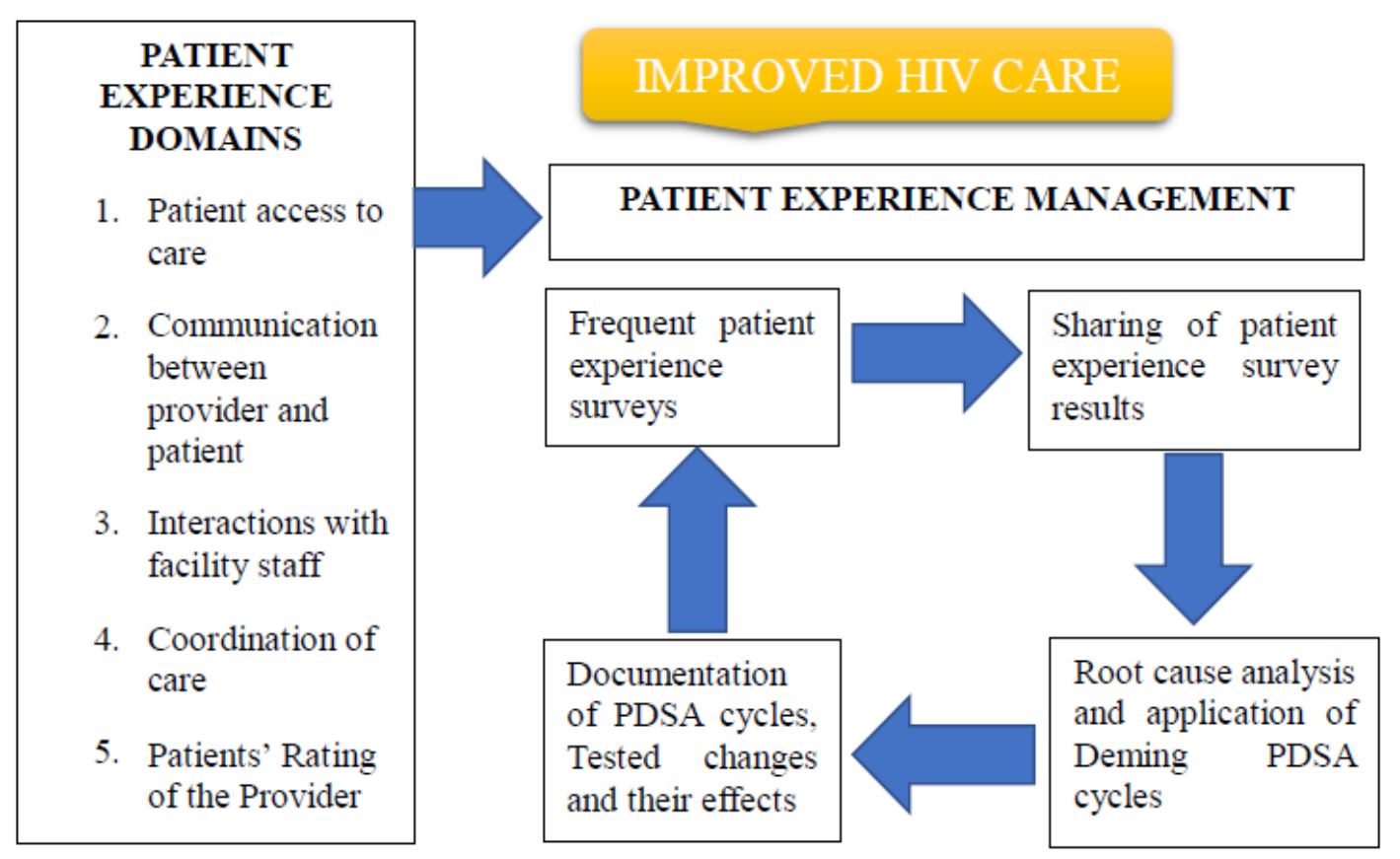

Figure 1. Conceptual Framework for Patient Experience Management to Improve HIV Care 


\section{Literature Review}

Globally, 36.7 million people are estimated to be living with HIV, and about 1 million AIDSrelated deaths occurred in 2016 alone, with SubSaharan Africa having more than half of the global burden. The UNAIDS 90-90-90 global targets were to accelerate progress such that, by $2020,90 \%$ of all people living with HIV are identified, of which $90 \%$ are put on Antiretroviral Therapy (ART) and of which 90\% are virally suppressed [5]. The Uganda Population-based HIV Impact Assessment (UPHIA) 2016/2017, [6] showed that the HIV prevalence had reduced from $7.3 \%$ as per the Uganda AIDS Indicator Survey 2011 [7] to 6.0\% among adults of 15 to 49 years of age. The report also estimated that 1.2 million people aged 15 to 64 and 95000 children below 15 years of age were living with HIV [6]. It is estimated that $62 \%$ of HIV positives were already in care [8] but only $56 \%$ were on treatment by June 2016 [9], of which by 2017 , only $45 \%$ and $65 \%$ were males and females, respectively [10].

\section{Adherence to Treatment, Appointment Keeping and Viral load Suppression among HIV Positives}

The success of HIV treatment is dependent not only on access to ART but also on retention in care and good adherence to treatment to achieve viral suppression, prevent virologic failure, reduce viral transmission, and HIV/AIDS-related deaths [11, 12]. The optimal adherence to treatment to achieve viral suppression is noted to be $95 \%$. However, in a study conducted in India, it was observed that 9.5\% of patients had poor adherence to HIV treatment of less than $80 \%$ [13]. In a study to determine the prevalence of adherence to treatment, patient self-reported adherence to HIV treatment of $50.4 \%$ was noted in Nigeria, associated with busy schedules of patients, simply forgetting to take their pills and religious constraints as major challenges to adherence [14].
Retention in care is one of the important indicators of a good HIV care program. A recent report by Uganda Virus Institute noted that only $50.2 \%$ of patients were retained on HIV treatment 12 months after ART initiation in 2018. It also observed that $26.6 \%$ of clients got lost from care during pre-HIV treatment in both rural and urban HIV clinics [15]. One study revealed an overall retention rate of $51.1 \%$ and a loss-to-follow-up of $52.9 \%$ in the first 6 months of ART initiation [16]. However, the lost to follow-up of clients in HIV care for newly enrolled pregnant and breast-feeding women was observed to be only $8.1 \%$ [17]. Retention of HIV clients in care is determined by regular and scheduled visits by the patients, and it is affected by missed appointments [18]. It is also important to note that adherence to clinic appointments is a predictor to adherence to treatment $[19,20]$. Studies have also shown a positive association of missing appointments with poor adherence to HIV treatment [21], failure to suppress viral load $[22,23]$, and drug resistance $[24,25]$. Other implications of missed appointments were noted to be increased costs in service provision and access and wastage of time for both the service provider and the client [26]. A 7\% of HIV clients in care missing their appointment in the last three months was observed [27]. Relatedly, a study in three settings of Cameroon observed that $49 \%$ of children in HIV care missed their appointment schedules, and this was attributed to longer follow-up appointments [28]. Contrary to that, only $1.8 \%$ of scheduled clients in a district-based hospital in Uganda missed their visits for refills in a period of 28 weeks of follow-up study [29]. The Uganda virus institute report 2018 noted that appointment keeping is one of the early warning indicators of HIV drug resistance being monitored by looking at the proportion of patients picking up all prescribed drugs on time before the previously prescribed and dispensed drugs have run out. The report further noted that $65.2 \%$ of patients keep their appointment at least within two days after the appointment date [30]. 
The ECASA Group of Consultants LTD in the Uganda AIDS Country Progress Report estimated that by June 2018, $87 \%$ of people living with HIV and on treatment had viral suppression [31], which results were close to the $76.8 \%$ [32]. It was also observed that the likelihood of having viral non-suppression remained significantly lower among adolescents who had been on ART for more than 9 years [32]. A study conducted on 219 individuals in HIV care observed a $33.9 \%$ undetectable viral load [33], a 70\% re-suppressed after adherence interventions [34]. The re-suppression was noted to be only $23 \%$ after 3 sessions of intensive adherence counselling among children under 20 years of age [35]. Viral load monitoring to clients on treatment 12 months after initiation revealed $79 \%$ coverage [36].

\section{Patient Experience and Management Indicators}

\section{Patient Access to Care and Appointment Scheduling}

Patient access to health care means having "the timely use of health services to achieve the best health outcomes" [37]. Good access to care is defined by the undelayed but easy gaining of entry into the health care system and getting access to sites of care where patients can receive the needed services [38]. Patient appointment mechanism directly determines the "timeliness" of the health care service given [39] while scheduling for health care services is a complex issue that requires the balancing of clinical process, patient needs, and influencing the capacity of health systems to offer appointments in a timely manner [40]. Efforts to patient retention in care should be based on the measures to maintain the provider-patient relationship and to understand the nature of patients falling out of care. Maintaining an appointment list and tracking whether patients attended the clinic is one of the ways to improve appointment keeping. This approach is said to allow for a more systematic look at the group of patients who don't keep the appointment, so that resources are targeted to engaging them [41]. Generation of a list of patients who missed an appointment on a particular day is one of the strategies for tracking appointment keeping and reducing on the time lag between missed appointments and when efforts are put to bring the patient back to care [26]. The fact that missed appointments cost both time and revenue, application of a systems-thinking approach, improving the flow of work and removing wasteful processes, redesigning of clinic workplace, and formation of a multidisciplinary team, have been observed to improve appointment scheduling [40]. Studies have shown that using a mix of approaches such as pre-appointment reminder calls, regular updates of patients' phone numbers and addresses, and phone calls after a missed appointment improve on patient appointment keeping [42]. Peer to patient's relationship was noted to reduce facility-based barriers to appointment keeping [43] and provide targeted psychosocial support services to reduce missed appointments [44, 45].

\section{Waiting Time}

Patient waiting time remains a problem in today's healthcare systems because of the dynamic, complex, and emergent nature of healthcare systems, as patients frequently have negative experiences with healthcare waiting rooms [39]. Reduction of waiting time of patients needs a systematic breakdown of workflow processes and identify problem areas [46, 47]. It is acknowledged that addressing waiting time is complicated as multiple stakeholders and operational processes are involved and interlinked. Delivering the best patient care was noted not to only rely on the clinical expertise of the health provider but also involves the interplay of the clinical setup, workflow, and communication. A project to improve the patient waiting time with revision of the appointment scheduling, patient reminders, more effective communication, and work reassignments, showed no improvement in waiting time due to fluctuating manpower, 
resources, and poor handover of patients from one provider to another within a visit but an increase in the percentage of patients seen by the doctor within a period, improvement in staff satisfaction and communication. However, poor appointment scheduling was noted to be a key component that contributes to delays and long waiting times for patients in outpatient clinics [47].

\section{Knowing where to Access Services and Communication between Provider and Patient}

Health care services are considered accessible when patients face no actual or perceived barriers to receiving services. It has been observed that supporting access to health care from the users' perspective helps patients to receive the right care at the right place and time. Integration of services, increasing staff numbers, expanding service delivery hours, reducing waiting times, and having systems for triaging patients, have been noted to improve access to care [48]. Communication between provider and patient aims at finding providers who meet the needs of individual patients and with whom patients can develop a relationship based on mutual communication and trust [38]. The domain is measured based on aspects of service provider listening carefully to the patient, explaining things in a way that is easy to understand, having respect for the patient, allowing enough time during the consultation, and providing adequate privacy during service delivery.

\section{Respecting what Patients say, Consultation Time and Service Provider Communication Skills}

While excellent clinical care remains the expectation, health care consumers are now seeking health care and supporting systems that respect patients [40]. It is observed that there is a need to involve patients in their own health and care such that service providers provide what matters to the patient, who are the experts of their own care. Shared decision-making was noted to be the process in which people who experience a change in their health work together with clinicians to select tests, treatment, management, and support packages [49].

The mismatch between the service provider and the patient during consultation, as the service provider is interested in making a diagnosis, while the patient focuses on the future actions of the service provider, is noted to exist [36]. It is, therefore, important that consultation meets the intentions of both the service provider and the patient. In a study to assess patients' satisfaction with a consultation at a general clinic in Saudi Arabia, it was observed that 53\% of the respondents were dissatisfied with it [50].

Patient experience has a series of complex healthcare processes, each comprised of numerous critical points of interaction between patients and the service provider. In a study to improve service provider's communication skills, the service provider's interpersonal skills were noted to be the most important in-patient experience. Patient satisfaction study of trained service providers and communication skills revealed that the proportion of "excellent" ratings of provider services increased, most notably; in the involving of patients in care decisions, explaining medical condition, the providers knowing the patient as a person, listening, and overall quality of care from the provider [51].

\section{Understanding Service Providers' Instructions and Interactions with Facility Staff}

It was observed that many patients leave their healthcare visit unsure of what their providers asked them to do or what was discussed, as only $12 \%$ of adult Americans have proficient health literacy, and only $30.1 \%$ of them could adequately teach-back [52]. It is known that other staff in the facility can influence the outcome of the patient's visit to the facility.

This is aimed at seeing other facility staff being supportive and helpful to patients, 
courteous, and respectful to the patients seeking care from the facility. Nonclinical and clinicalsupport personnel have a powerful influence on how patients perceive their entire care experience, just like the medical practices can improve the overall care provided by focusing on nonclinical and clinical-support services to create a strong first impression of patients at every care visit, to prepare clinical staff to deliver excellent service, minimizing needless delays in service delivery, and prioritizing the services that patients consider to be most important [53].

\section{Coordination of Care and Knowing Important Medical History}

Coordination of care is a conscious effort to ensure that all key information needed to make clinical decisions is available to patients and providers [38], and it is defined as the deliberate organization of patient care activities between two or more participants involved in a patient's care to facilitate the appropriate delivery of health care services, [54]. Studies indicate that over 80 percent of diagnoses are based on medical history [55], a powerful diagnostic technology [56]. It is suggested that a more rational approach to the use of the medical history led to improvements in diagnostic efficiency and effectiveness, with benefits for individual patient care [56].

\section{Medication Information}

Patients are reported not to understand the instructions they receive on medications, and 40-80 percent of the medical information communicated by health care practitioners is completely forgotten by the time they reach home [57]. Patients always have challenges in taking their medications as a result of; limited patient empowerment, mistrust of prescribed medications, and frequent noncommunication about medications prescribed. It was also noted that patient-centered care makes service providers view patients as partners in their disease management and therefore talk to patients about the prescribed medicines [58].

\section{Methodology}

The study was conducted in Jinja district Uganda, at Bugbee, Budondo, Walukuba, and Mudede health centres, purposively selected due to their high patient numbers. The study units were files of adult HIV clients identified with any of the poor HIV care outcomes of; missed appointments of $>$ two weeks, poor adherence to treatment of $<85 \%$, and unsuppressed viral load of $>1000$ viral copies per milliliter. Duration in care was one of the determinants for a client file to be considered for the study, as only clients who have been in HIV care for at least 6 months are eligible for a viral load test by the Uganda Ministry of health standards. Patients of the selected files above were also targeted for an interview as study units.

Lastly, the PDSA cycles at the study health facilities were also used as study units during the study. The sample size estimate of 226 was determined by the use of the hyper geometric method for a known population. Files of HIV active clients for the period of October to December 2018 were reviewed to identify any of the study HIV care outcomes, of which 437 were found to fit in the inclusion criteria. 224 clients for the identified files were interviewed at baseline in January 2019 and end-line assessment in July the same year.

The action research involved interventional, observational, descriptive, and qualitative study methods. Data collection was done in three parts; 1) the client-patient experience exit interviews using the Customer Assessment of Healthcare Providers Systems (CAHPS). This experience was determined by 14 elements on a Likert scare of 'Never,' 'Sometimes,' 'Usually,' and 'Always,' and 1 element of rating service providers on a scale of 1 to 10 , with 1 being the lowest, 2) the patient HIV care outcomes, and 3) the PDSA cycles, using the documentation journals. Monthly quality improvement 
meetings were held to share results and experiences among the study health units.

The data was analyzed using Microsoft excel, and results were shared with the 4 teams of health unit managers to conduct a root cause analysis of the findings at baseline and plan for improvement using journals to document the PDSA cycles. The baseline and end-line assessment findings are presented in tables and narratives with percentages of the various parameters of client experience and quality of care.

From the Likert scale of the patient experience measured indicators, results in percentages of respondents were analyzed and presented. Findings from the PDSA cycles were presented in a descriptive manner to give a picture of the interventions at the study sites. The study followed the professional codes of conduct for research, including observing subjects' right to privacy, confidentiality, and informed consent.

The study protocol was approved by Milldam Uganda Research and Ethics Review Committees (REC REF 0309-2018), and the Uganda National Council of Science and Technology (UNCST) (HS 2510). I declared not having any conflict of interest or relationship with the study sites, research, and ethics committee, and they had no influence in any manner that might have affected the validity of the study.

\section{Results}

\section{Demographics of the Study Client Files}

The study showed that $29.3 \%$, and $70.7 \%$ of client files identified with any of the poor HIV care outcomes were for males and females, respectively. The prevalence of the study HIV care outcomes at baseline was $13.6 \%$, with Bondo, Bugembe, Mpumudde, and Walukuba health centre having $7.3 \%, 14.8 \%, 13.4 \%$, and $17.9 \%$, respectively.

Most of the client files included in the study were for clients who had been in care for more than four years.

\section{Results on the HIV Care Outcome Indicators}

The findings show that a total of $52(11.8 \%)$ client files were identified with poor adherence to treatment at baseline assessment. Walukuba health centre having only $0.7 \%$ of its clients with a poor adherence score at baseline is uncommon, and it is possible that the assessment for adherence for the HIV clients in care was being done wrongly by the service providers, as at endline assessment, it was observed to be at $11.3 \%$. The study revealed that 285 (65.2\%) of the study files had more than two weeks of missed appointments, and a reduction to $88(25.1 \%)$, at end-line assessment. It was also observed that of the 285 client files of missed appointments for more than two weeks, 266 (93.3\%) had an adherence score of $>85 \%$ at baseline assessment.

The study revealed that at baseline assessment, 203 (46.5\%) of study client files were due to unsuppressed viral load, which reduced to $109(31.5 \%)$ at end-line assessment. It was also revealed that $142(70 \%)$ of all client files with unsuppressed viral load were for clients who had been in care for more than four years. $162(37.1 \%)$ of client files studied had undetectable viral load at baseline assessment, which improved to $181(51.7 \%)$ at end-line assessment. $34(7.7 \%)$ of the study files had never had a viral load test done to the clients at baseline assessment, which reduced to 5 (1.4\%) at end-line assessment. 30 (83.3\%) files of clients who have never been done a viral load at baseline assessment were between 6 months and 2 years in care. It was observed that 151 (34.5\%) of the study client files were overdue for a viral load test to be done at baseline assessment, which reduced to $59(16.8 \%)$ at end-line assessment. The study further showed that 192 (91\%) client files with unsuppressed viral load had adherence to a treatment score of $>85 \%$ at baseline assessment, which reduced to 47 $(42.2 \%)$ at end-line assessment. This could be pointing to resistance to HIV treatment, which is a challenging situation. 


\section{Results on the Patient Experience of HIV Care at the Study health Facilities}

The study identified 437 potential respondents based on the study selection criteria, out of which 224 were interviewed at both baseline and end-line assessments. Specifically, there were 44, 82, 45, 43 respondents for Budondo, Bugembe, Mpumudde, and Walukuba health centres at baseline, and 52, 64, 51, and 57 at end line assessments, respectively. For patient access to HIV care, there was an improvement on the percentage of respondents reporting that they "Always"; get appointment schedules from service providers, access HIV care service within four hours from arrival to exit and get to know where to access HIV care services within the health facility, from an averagely of $44.9 \%$ to $86.2 \%$. Secondly, there was an improvement in communication between service providers and the patients from the average of $57.6 \%$ to $92.4 \%$ of the respondents reporting that they are "Always"; listened to by the service providers, explained to in a way that is easy to understand, showed respect for what they say, given enough time during consultation, and adequate privacy during service delivery. The study results also showed an improvement in the patient experience domain of interactions between the patients and the facility support staff from an average of $54.8 \%$ to $88.4 \%$ of the respondents reporting that the support staff are "Always"; helpful, courteous, and respectful to patients. Fourthly, there was an improvement in the coordination of the HIV care from an average of $37.2 \%$ to $85.4 \%$ of the respondents reporting that their service providers are "Always"; knowing important information about the patients' medical history, getting someone from the service provider's room to follow patients up to the next service point, providing patients with all the prescribed service packages, and talking to patients about all the prescribed medicines. Lastly, the patients' improved satisfaction with the service provider for the services received from $28.3 \%$ to $50.5 \%$ of the respondents scoring "Excellent".

\section{The Effect of Application of the Deming PDSA Cycle as a Patient Experience Management Tool}

\section{Appointment scheduling in HIV care}

During the implementation of the study, it was observed that appointment keeping was a challenge, especially for Bugembe, Walukuba, and Mpumudde health centres. Patients used to come in any day and time for treatment, and anyone at the facility could prescribe and refill ART for the clients. At Mpumudde, some of the support staff, like the linkage facilitators, could at times supply more drugs than what is prescribed, and there was no harmonization of appointment dates with the drug supplies. Completing the HIV client card was a challenge to almost all the health centres, and it could be hard to know that important information or service offered to a client. Health managers were not concerned about what was happening in the clinics, and the linkage facilitators were serving clients out of sympathy. During the implementation of the project to improve on appointment keeping at all the study health facilities, health managers realized that they had left the leadership of the HIV clinics to lower cadres, and the quality of care was noted to be limited. At Mpumudde health centre, several meetings were held with the health facility managers to support them realize the areas of weakness, and this helped in a number of ways, including the allocation of more staff to the clinic. Using the Deming PDSA cycles, the following tested changes were used; all the study health facilities conducted harmonization of appointment date with drug supplies through pill counting of drug balances for the client to ensure that a client is supplied only enough for the next appointment date. The health managers also defined the roles of linkage facilitators to ensure that they do not prescribe or dispense drugs to clients. HIV clinic days were harmonized, and all clients were informed of the changes in the HIV clinic days such that other days were only for new HIV clients or those with complications 
to treatment. The creation of a box or space for files on appointments helped in the identification of those who have missed attending the clinic. This was followed by a phone call to the client who had missed the clinic the following day. Service providers also practiced on-spot documentation of the services offered to the clients in the HIV care card to avoid incomplete information capture, which could affect clinical decisions.

\section{Reducing Waiting Time for Patients during HIV Care}

Waiting time was noted as one of the major challenges being faced by HIV patients as they accessed care, especially at Walukuba, Bugembe, and Mpumudde health centres. The HIV clinics at the study sites were understaffed, yet the few service providers used to start clinics late, there was no order of service of first come, first serve, the laboratory services were a distance from the clinical rooms, and patients used to get lost within the facility. All these gaps were contributing to delayed access to services and, therefore, long waiting times for the patients. A number of change ideas were applied using the PDSA cycles to improve on the patient waiting time as follows; change in the client flow to reduce on unnecessary movements of both the clients and the service providers, provision of services in a one-stop centre for easy access to all the services, provision of fast come fast serve card to enable the early comers to access services first, increasing staff to provide services, identification of files for patients on appointment a day before the clinic day, pre-parking of drugs for clients before the clinic day, and ensuring early starting of the clinics. These practices were scaled to all the study health facilities, and they realized improvement in the patient experience with waiting time.

\section{Increasing Access to Services within the Clinic}

The service providers of Walukuba and Bugembe health centres were surprised to realize that some of their clients were not aware of where to access all the prescribed services. This was because there was one waiting area for all outpatients, which caused stigma and confusion to patients as to which room or service provider to see. The rooms for provision of services were not labelled for easy identification of the service sites, and service sites were distant, especially the laboratory and the pharmacy, and limited communication to patients on where to access the different services within the health facility. In an effort to improve the situation, and using the PDSA cycles, the service providers identified safe spaces for the HIV clients where all the services were provided during particular clinic days, and such spaces were also used for other services during non-HIV clinic days to reduce stigma. There was also labeling of the service points within the service areas for easy directions to the clients. Walukuba health centre, positioned the records assistant at the registration point to direct all clients to the next service point after registration.

\section{Listening Carefully to Patients Concerns}

During the study, clients of Walukuba and Bugembe health centres reported not being listened to "Always" by the services providers. The health managers attributed the situation to the allowing of support staff with limited training and skills to provide HIV care, uncoordinated patient appointments, and the poor leadership and management of the HIV clinics as service providers had organized absenteeism where a few service providers would be available for duty compared to the expected. The study supported the engagement of the health manager to create clinic days for the different categories of clients (differentiated HIV care) notably, the children, the adolescents, and the virally unsuppressed clients. Identification of a nurse to do triaging of HIV clients during clinic days such that all stable clients are served fast, and only the unstable clients could see the clinician, allocation of more staff during the clinic days to reduce the work 
pressure, and harmonization of appointment schedules were the strategies implemented to improve on the patient experience of being listened to during their visit to the service provider.

\section{Explaining Things to Patients in a Way that is Easy to Understand}

During the study, Walukuba health centre was reported to have service providers who do not always explain to patients in a way easy to understand during the baseline assessment interview compared to other study health facilities. It was noted by the health managers that Walukuba health centre had linkage facilitators who had little knowledge on some of the medical terms, yet they had much more interface with the patients than the quantified staff. It was also noted that the facility is in a community with mixed tribes and languages. The facility gets clients who do not understand the commonly used local languages in the area, and the service providers were not able to speak to them, hence the need for an interpreter. These factors affected this patient experience element, and in a way to improve, the health facility managers allocated more qualified staff to the HIV clinic to reduce the interface of the patients with the support staff. The managers also allocated a linguistic linkage facilitator to interpret for patients. The two interventions improved the situation at Walukuba health centre as the 'Always' response by the study participants on whether the service providers 'Always' explained things in a way and language easy to understand increased from $24.5 \%$ to $98.2 \%$ at baseline and end-line assessments respectively.

\section{Service Provider Respecting What Clients say during HIV Care}

Respecting of what patients say by the service provider is a right, and it maintains the dignity of the patient. During the study, respect for what clients say by the service providers was good at most of the study health facilities except at
Walukuba. Walukuba health centre service providers reported not having been aware that patients were not feeling comfortable with the limited respect of what they say during access to care. The findings prompted the health managers to hold a meeting to give feedback to all the service providers. It was also agreed that the service providers implement the communication of the patient experience rights to the clients during every clinic day. Patients gained the confidence to express their dissatisfaction during the exit interviews, and such feedback was being shared during the monthly quality improvement meetings. The study showed an improvement in the "Always" response to service providers showing respect to what patients say most, especially at Walukuba health centre, from $24.5 \%$ to $96.5 \%$ at baseline and end-line assessments, respectively.

\section{Service Provider Spending Enough Time with Clients during Consultation}

It was a fact that all the HIV clinics were understaffed and overcrowded as the facilities were running only one clinic day in a week. Under such a situation, it was hard for the service provider to spend enough time with the patients during consultation.

The interventions were an additional human resource to the clinics, the orientation of the new staff on the key aspects of the clinic running, and the splitting of the HIV clinics in a differentiated manner as the unsuppressed clients, children, and adolescents were being seen during their special clinic days. Bugembe health centre being with the biggest number of clients also split the general HIV clinic to run for two days in a week. These interventions worked for the health facilities and there was improved time for consultation.

\section{Improving Privacy for Clients during HIV Care}

HIV is a very stigmatizing and discriminating disease in Uganda, and this has affected access and utilization of HIV services. Privacy at 
Budondo and Walukuba health centres was grossly rated low by the majority of the respondents. This was attributed to the fact that general outpatients were mixed with the HIV clients during access to care. It was also a practice at Budondo and Walukuba health centres that HIV client files would be read by name in front of all the outpatients. It was also possible to see and hear the details of the service provider's discussion with the HIV client at Budondo health centre as the window for the clinician's room had no canteens, and the service point was just near the window.

At Mpumudde health centre, the sorting of files, recording, intensive adherence counselling sessions and dispensing was being done in the in-patient ward. All the in-patients were seeing and hearing all that was taking place. At Bugembe and Walukuba health centres, dispensing of drugs for the HIV clients was happening at the general dispensary, and it was hard to observe the privacy of the HIV clients. All these factors have compromised privacy and increased stigma at the health facilities. It had resulted in patients coming on non-clinic days and late in the evening when many of the outpatients have left the facility.

It also affected appointment keeping, adherence to treatment, and access to other services like family planning. At Budondo health centre, a new room was allocated for HIV care. The room was near the filling room for easy transfer of files and storage. Budondo and Walukuba health centres piloted the use of first come first serve cards with numbers. This eliminated the calling of clients by name to access services. Walukuba, Bugembe and Mpumudde health centres made the HIV clinics a one-stop centre with almost all the services in one area. The space for the clinic was closable for the general patients not to know what is happening in the clinic. This was a magic bullet to privacy as it reduced stigma improved the client's confidence, expression, and access to HIV service.

\section{Support Staff Helping and Respecting Clients during HIV Care}

Access to HIV care can be affected by the communication of the support staff to the patients. Lack of help and respect to the clients seeking care by the support staff creates a bad experience of the care. It is important that support staff are helpful and respectful to the clients seeking care to contribute to a good experience with the services received. At baseline assessment of patient experience, Walukuba and Bugembe health centres had lower percentages of the "Always" response of $24.4 \%$ and $50.9 \%$ compared to Mpumudde and Budondo health centres with $78.8 \%$ and $75.6 \%$, respectively. The health facility teams attributed it to the fact that the HIV clinics at the affected health facilities were mainly being run by the linkage facilitators, who are support staff. The linkage facilitators had taken on the roles of the qualified staff, yet they had no professional ethics to practice. At Bugembe health centre, the health managers redefined the roles of the linkage facilitators and limited their involvement in the roles of the qualified staff. At Walukuba, one of the notorious linkage facilitators was replaced with another one, and this improved the situation. It is also true that the addition of more qualified staff to the clinic also reduced the interface of the linkage facilitators with the clients. Budondo and Mpumudde also practiced the redefining of roles for linkage facilitators through meetings that involved the health managers, the HIV clinic service providers, and the linkage facilitators. It was also observed that the support staff became more respectful and courteous to patients during access to HIV care at all the study health facilities.

\section{Service Provider Knowing Important Information about the Client Medical History}

Studies indicate that over 80 percent of diagnoses are based on medical history. Reviewing and knowing the past medical history of an HIV client in care helps in the provision of 
quality HIV care services. It was revealed that the HIV clinics in the study health facilities were inadequately staffed, and this was affecting the time required for the service provider to review client cards to know important information of the client's medical history. It was also noted that the HIV service providers were not stable as health managers could rotate and switch service providers to different departments at any time. It was also true that the highly qualified and senior service providers were not interested in working in the HIV clinics, and they were also not attending refresher training on HIV care. At all the study health facilities, the nurses and linkage facilitators were the ones mainly managing the clinics. This was affecting the quality of HIV care provided and therefore making it hard for the service providers to review and have an adequate medical history of the clients under their care. The magic bullet to this problem was increased staffing into the HIV clinics and engagement of the health managers to allocate appropriate carders of service providers to the clinics. For example, Mpumudde allocated a clinical officer, a laboratory technician, and a nursing officer to the clinic, while Budondo allocated a medical officer, a clinical officer, and a nursing officer. Bugembe acquired two more nurses, while Walukuba added a nurse and a laboratory staff to the clinic. The strategy was noted to reduce the chances that a lower carder manages the HIV clinic. The strategy improved the "Always" response to service providers knowing important information about the patient's medical history above $90 \%$ at all the study health centres.

\section{Follow up of Clients to Next Service Points during HIV Care}

The study noted that follow-up of clients to the next service point was only being implemented by Mpumudde health centre, which attributed it to physical escort of the client to the different service points by the linkage facilitators. Other study health facilities also had linkage facilitators, but they were not being used to follow up clients to the next service points. It was also reported that the facilities were understaffed to have someone follow up on clients to the next service points. Bugembe, Walukuba, and Mpumudde health centres opted to have a one-stop centre for almost all the services such that the next service points are within the same room or area, and it was not necessary to escort a client but to direct him or her to the next service table by pointing. Budondo health centre did not opt for any of the interventions, and this affected their improvement on this element.

\section{Providing all the Prescribed Service Packages to Patients during HIV Care}

The problem of providing limited prescribed service packages almost affected all the study health facilities. The service packages that were noted by the health facility managers as affecting this element were drugs, and Viral load testing. Budondo, Walukuba, and Mpumudde health centres had a low viral load coverage as some patients for this service were waiting for long hours at the main laboratory. It was realized that some clients would leave the laboratory without getting the service but convince the dispensing staff that they have been bled for the test to access their drugs. Bugembe health centre had solved this through the engagement of the laboratory staff to give priority to clients for viral load, and the strategy was working for them. This is what Budondo health centre tried, and it also worked for them. Walukuba and Mpumudde health centres innovated to have the viral load bleeding site within the HIV clinic to avoid missed opportunities, and this was a very successful strategy for them. On the side of drugs, there was always adequate stocks of ARVs, and access was ensured. However, the facilities had limited supplies of drugs for opportunistic infections, especially Cotrimoxazole. Budondo health centre had enough of drugs for opportunistic infections as it was practicing good procurement and rationing of drugs. Walukuba and Bugembe health centres 
tried the same strategy, and it also worked for them. Mpumudde did not succeed as the mandate to order and store drugs was not in their hands, and the in-charge was not willing to support this arrangement. It can therefore be concluded that working as a team with the laboratory staff either to give priority to clients for viral load in the main laboratory or creating a bleeding site in the HIV clinic improves viral load coverage and access.

\section{Talking about the Prescribed Medications to Patients during HIV Care}

The knowledge of patients on the medications provided increases adherence to treatment and improves patient's participation fully in their care. The service providers agreed with the study results that they were not giving adequate information to clients about the prescribed medicines. This was attributed to the thinking that clients were familiar with the drugs they take, yet at times there was a change in the size, package, and color of the medicines. There were also instances where substitution and switching of drugs was being done but still with no adequate information given. This was affecting adherence to treatment, and at times clients would take from one type of ARVs that they are familiar with and leave the ones they are not familiar with. The health managers of Bugembe and Mpumudde health centres had not assigned a qualified staff to dispense instead, it was the linkage facilitators (support staff) dispensing. This could affect the level of information about medicines that could be given to the clients. At Walukuba health centre, dispensing was being done at a general pharmacy where all patients were getting medicines from, and health workers were too busy to give enough information to patients, coupled with the stigma that patients were facing during the dispensing time as HIV drugs are unique and packaged in plastic bottles. The same situation was at Mpumudde health centre as the dispensing window was facing the in-patient ward, and all in-patients would see and hear the instructions to the HIV clients during dispensing. To improve on the patient experience element of talking about the prescribed medicines, Walukuba and Mpumudde health centres made and implemented a health talk program on medicines during each clinic to remind clients on the drugs they take but also to give new changes in the HIV care and treatment. Bugembe and Mpumudde health centres put up a fulltime nurse to dispense drugs including giving full instructions on the medicines. There was a change in the dispensing area at Walukuba health centre as they transferred the HIV drug dispensary into the HIV clinic room to make HIV care a one-stop centre, while Mpumudde health centre resorted to dispensing within the dispensing room to reduce stigma during dispensing.

\section{Rating of Service Providers}

Picking an opinion on how the patient rates a service provider gives a picture on whether the patient appreciates the efforts of the service provider in providing a service, and it is also an indicator that the patient will adhere to the service provider's instructions or even come back for services next time based on the built trust. It is a measure of how satisfied the patient is with the services of the service provider. The study showed an improvement in the satisfaction rates with the service providers across all the health facilities.

\section{Discussion}

\section{HIV Care Outcomes of Adherence to Treatment, Missed Appointments and Viral Suppression}

The study revealed that females identified with poor HIV care outcomes were two times more than the males. This could be due to the fact that there are more HIV-positive females (65\%) in Uganda accessing HIV treatment than males $(45 \%)$ [10], even when the study did not determine the percentages of males and females on treatment at the study health facilities. It is therefore important that efforts should be geared 
towards men accessing HIV treatment in the study health facilities. Retention in care is important for a good HIV care program. The study showed an $80 \%$ retention in care during the 6 months of study. This was higher than the $50.2 \%$ retention on HIV treatment 12 months after ART initiation [15], respectively. The difference could be due to the periods of study, i.e., 6 months and 12 months in the two studies.

There was a $10.3 \%$ lost to follow during the 6 months of study, compared to the $26.6 \%$ observed among pre-HIV treatment clients in care at both rural and urban HIV clinics [15]. The findings are similar to the observed lost to follow within 6 months of treatment initiation that reduced as patients stayed longer in care [16]. However, it was observed that among pregnant and breast-feeding women, the lost to follow among newly enrolled HIV positives to treatment was as low as $8.1 \%$, [17]. Conclusively, the longer a patient stays in care, the less likely that he/she will get lost to followup, and therefore, the need for many efforts in early than late retention in HIV care.

The researcher did not come across data on the death rates of HIV-positive individuals in health facility clinics but observed a $1.3 \%$ death of the study clients during the 6 months period of study. This confirmed that retention in care is affected by death, among other factors. It was also hard to get data on the rate at which client files get lost with in the health facilities, which affects the quality of HIV care as the history of the patient cannot be traced. The study revealed a $3.4 \%$ loss of files for clients studied within a period of 6 months. The loss of client files reflects a poor filling and patient information management system, and service providers need to come up with strong interventions to reduce on the loss of client files and records within the health facility.

There was no literature accessed on the possibility of having a poor HIV care outcome despite being in care for a longer duration. However, the study showed that more than 55\% of clients identified with a poor HIV care outcome mostly unsuppressed had been in care for more than 4 years. It can therefore be concluded that being in HIV care for a long time does not guarantee good HIV care outcomes. It is recommended that service providers continue supporting all clients to adhere to HIV treatment irrespective of their duration in care.

\section{Adherence to HIV Treatment}

Adherence to HIV treatment is very key in suppressing the viral load, and studies have shown a positive association of missing appointments with poor adherence to HIV treatment [21]. The study revealed that $11.8 \%$ of all the clients identified with poor HIV care outcomes had a poor adherence of $<85 \%$. The finding is almost similar to a study conducted in India that observed that $9.5 \%$ of patients had poor adherence to HIV treatment of less than $80 \%$ [13]. The study also observed that $93.3 \%$ of patients who had missed an appointment for more than two weeks were scored an adherence level of $>85 \%$. This is ridiculous, and it can be attributed to the knowledge gap and or noncommitted service providers to score patient adherence to treatment per national guidelines. The following change packages were used to improve adherence scoring and patient adherence to treatment using the Deming PDSA cycle; Mentorship to service providers on patient adherence scoring and documentation, Intensified health education and information on drugs supplied, and intensive adherence counseling to all patients with poor adherence. The interventions were noted to reduce poor adherence to treatment. Therefore, it is recommended that the above interventions, when implemented as a package, improve service providers' capacity to score adherence and patient adherence to treatment.

\section{Adherence to Appointment Schedules in HIV Care}

It is important that patients keep their appointment schedules if adherence to treatment is to be ensured. The study revealed that $65.2 \%$ 
of the studied client files at baseline were due to missed appointments for more than two weeks in the previous three months. The period of missed appointments for at least two weeks in the studied client file was taken by the researcher to cater for such common practices of service providers not taking into account of cumulative pill balances from previous patient refills. The findings were close to the observed 49\% [28], but too high to the $7 \%$ of [27]. The difference could be as a result of the differences in the period of review for the status of missed appointments, the duration of the missed appointment, and the age category of clients studied. During a root cause analysis of the causes of missed appointments at the study sites, the service providers identified that clients were having cumulative drug balances during their visits, and service providers were not doing pill counting to ensure that the drugs given balance with the date of appointment. The cumulative balance could make patients not keep appointments since they would not see the reason for coming back to the health facility. The clients were therefore being scored with good adherence, yet they did not keep the appointment as evidenced by the $93.3 \%$ who missed appointment but were scored a good adherence across all the study facilities at baseline. The service providers also observed that some clients were coming for treatment any time and day, including weekends, and this could affect the documentation of the services provided in the client record cards and registers as some working spaces and individuals may not be available or accessible. The third factor that was affecting appointment keeping was that some clients would get excess drugs through the health facility support staff without documentation and consent of the technical service providers. Lastly, there was a systemic failure of timely documentation and update of the client records. All these were the service provision challenges on adherence to appointment schedules across all the study health facilities. There was no literature accessed by the researcher that noted the above as some of the challenges to appointment keeping, but the available information was related to patient and community factors only.

To improve appointment keeping, the project used a systems-thinking approach to improve workflow, redesigned the clinic workplace, and used a multidisciplinary team [40] because using a mix of approaches such as pre-appointment reminder calls, regular update of patients' phone numbers and addresses, and phone calls after a missed appointment improve on patient appointments keeping [42]. The change package used to reduce on missed appointments were Pill counting to harmonize the pills supplied with the appointment date, Emphasis on strict appointment keeping for patients and duty attendance of the service provider during the clinic days, post missed appointment follow up phone calls to patients, Timely recording and updating of the client information required, and Engagements of the health facility managers to increase staffing of the HIV clinics. However, the end-line assessment results did not show much reduction in the percentage of client files with both missed appointments of more than two weeks and an adherence score of $>85 \%$ from $93.3 \%$ to $60.3 \%$. This means that the intervention of mentorship to service providers on patient adherence scoring and documentation had less effect on adherence scoring and or oversupply of drugs compared to the appointment schedules. It is therefore recommended that the study sites need to review the problem to identify other root causes such as multiple site visits by the clients and develop interventions to close the gap.

\section{Viral Suppression in HIV Care}

The ultimate goal of HIV treatment is to achieve viral suppression among patients. The third ambitious target for Uganda as adopted from UNAIDS is to achieve a $90 \%$ viral suppression by 2020 among HIV clients on treatment. Poor viral suppression leads to increased chances of developing resistance to 
treatment, opportunistic infection manifestations, HIV transmission, and eventually death. Baseline assessment results showed $46.5 \%$ of the client files identified with any of the study poor HIV care outcomes being due to unsuppressed viral load of $>1000$ copies/ml. The results do not match with the $13 \%$ unsuppression among the total clientele of the HIV clinic [32], compared to study results based on clients who had any of the poor HIV care outcomes studied. The findings agree that re-suppression occurs after adherence interventions [34] [35], as it was found out that only $31.5 \%$ of the study client's files were still with unsuppressed results at the end line assessment. The study also revealed that only 7.7 of the study files had never been done a viral load test at baseline assessment, which reduced to $1.4 \%$ at end-line assessment. The viral load test coverage is in agreement with the $75 \%$ coverage in 2017 [31]. The study further revealed that $70 \%$ of all the unsuppressed client files studied were for clients who had been in care for more than four years. This may be interpreted that the more a client stays in care, the more likely it is that he/she will turn unsuppressed. These findings are contrary to those from Cambodia that stated that the likelihood of having viral non-suppression was significantly lower among adolescents who had been on treatment for more than 9 years [32]. The difference could be related to differences in the health care systems in the two countries but also in the age categories studied. The rate of undetectable viral load among the study files was only $37.1 \%$, at baseline assessment similar to the $33.9 \%$ undetectable rate for viral load [33]. The undetectable viral load rate among the study files at the end line assessment improved to $51.7 \%$.

The study also observed that $83.3 \%$ of the client files expected to have had viral load monitored, and with results, the respective HIV patients had never been done any viral load test between the period of 6 months and 2 years. The study further observed that $34.5 \%$ of the study client files were overdue for a viral load test to be done based on the Ugandan Ministry of health standards. However, this was reduced to $16.8 \%$ at end-line assessment. The findings at both baseline and end-line assessments are similar to the observed $21 \%$ of HIV-positive clients who were due for viral load 12 months after initiation to treatment but did not have it done [36]. The effect of the observed gap leads to poor HIV care as the right decisions are delayed to be made. Health facilities need to have a mechanism of ensuring that all clients due for viral load access the service. It is also common for patients who are due for viral load testing missing the service even when they make a visit to the clinic. The study also showed that $91 \%$ of client files with unsuppressed viral load had adherence to treatment score of $85 \%$ and above at baseline assessment, which reduced to $42.2 \%$ at end-line assessment. This could be pointing to resistance to HIV treatment, which is a challenging situation. During the study, it was noted by the service providers that efforts to address challenges to viral load suppression were limited. To note; there was limited access to viral testing, late return of viral load test results, documentation of the viral load results in the client care cards was inadequate, documentation of intensive adherence counselling sessions was not effective, and timeliness of the sessions was not as per the ministry of health standards that guide that each session should be conducted one month apart.

The change packages tested to improve viral load suppression were Assigning of staff responsible for viral load, creating a viral load bleeding area within the HIV care site, timely tracking of patients for viral load testing and results, provision, and documentation of intensive adherence counselling sessions to HIV clients with unsuppressed viral load. The intervention resulted into a reduction in the number of clients with unsuppressed viral load among the study clients, as at baseline assessment, there was $46.5 \%$ of the study client files with an unsuppressed viral load of $>1000$ 
copies $/ \mathrm{ml}$, which reduced to $31.5 \%$ at end-line assessment. It is therefore recommended that service providers need to implement the above strategies as a bundle to ensure re-suppression.

\section{Patient Experience Management}

Patient experience has not been used as a means of assessing the quality of care in Uganda yet, if implemented, it leads to a memorable event in patients. It is important to note that patient care requires multiple practitioners during service provision, causing communication differences variations in protocols and approaches. It is therefore important to note that patient experience is beyond the patient-clinician interaction but the whole health care system.

Patient Experience Management has been used to close gaps in patient care especially for chronic conditions like HIV, and it creates a model of attention focused on the patient experience to accomplish the "Wow" effect in patient's access to health care. There has been challenges in patient experience management before of resistance to change, other organizational priorities, lack of support from senior staff, and limited resources. There is limited literature on how patient experience management has been used to improve patient centered care.

Therefore, this study adopted the Deming PDSA cycle approach because it is the most commonly used quality improvement approach in healthcare, and it uses a rapid cycle process, the Plan Do Study Act cycle, to test the effects of small changes in a short time using a systematic and data-driven approach to implement, test, and sustain changes.

\section{Improving Appointment Scheduling and Reducing Waiting Time for Patients during HIV Care}

It was observed that appointment keeping was a challenge, and health facility teams used different interventions like; Harmonization of HIV clinic days and appointment date with drug supplies through pill counting, timely documentation of appointment schedules, line listing clients, and identifying those who miss appointments schedule in an effort to bring the patient back to care, and patient re-engagements and similar observations have been made before [26].

Scheduling for health care services requires the balancing of clinical process, patient needs, and influencing the facility health systems to offer appointments in a timely manner [40]. It was in a similar way observed during the implementation of the above strategies to improve appointment scheduling.

Waiting time was noted to be one of the major challenges faced by HIV patients as they accessed care and a number of change ideas that were used to improve on it include a change in the client flow, creation of a one-stop centre for HIV care, and increased staffing. Although the interventions above worked well for the study health facilities, they did not show improvement in waiting time but improved staff satisfaction and communication [47]. This could be attributed to differences in the settings under which the studies were conducted.

\section{Increasing Access to Services within the Clinic and Listening Carefully to Patients Concerns}

Health care services need to be accessible to patients at the facility without any physical or perceived barriers. During the study, it was observed that the waiting area for some outpatients were contributing to stigma to patients during access to HIV services. The rooms for provision of services were note labelled for easy identification of the service sites. The laboratory and the pharmacy were a distance away from the clinical rooms, yet there was limited information to patients on where to access the different services within the health facility. The following tested change packages were used to improve patient access to services within the health facilities; Provision of services in a one-stop centre, labeling of the service 
points for easy directions to the clients, Designation of staff to triage patients and direct them to the next service points.

The study showed that the majority of the HIV patients at Walukuba and Bugembe health centres were not "Always" listened to by the services providers. Listening carefully to patients by the service provider improves the trust of the patient that his/her medical and psycho-social issues will be well understood and taken care of. The interventions to the observed situation were Creating separated clinic days for the different categories of clients, notably the children, the adolescents, and the virally unsuppressed clients, additional clinic staffing to reduce work pressure, and harmonization of appointment schedules with the created clinic days.

\section{Explaining Things to Patients in a Way that is easy to Understand and Service Provider Respecting what Clients say during HIV Care}

Walukuba health centre was observed not to be explaining things to patients in a way easy to understand at baseline assessment as it had its support staff (linkage facilitators) with many interfaces with the patients than the qualified. The health teams at this facility implemented the following tested changes to improve on the situation; Additional staffing of the clinic to increase on service providers interface with patients and allocation of an interpreter for clients with languages not known by the service

\section{References}

[1] Moen D. Ronald and Clifford L. Norman, (2010). Circling back. Clearing up myths about Deming cycle and seeing how it keeps evolving. https://deming.org/wpcontent/uploads/2020/06/circling-back.pdf.

[2] Bita Kash1* and Molly McKahan (2017). The Evolution of Measuring Patient Satisfaction. Journal of Primary Health Care and General Practice. provider. The tested changes were in conformity with [36] observation that "excellent" ratings of the service provider increased when patients were involved in care decisions.

\section{Conclusion}

The improvement in the HIV care outcomes using patient experience management is hinged on improved health facility leadership and governance, skilled human resources, and client flow in a one-stop centre mode. The ministry of health needs to support the implementation of patient experience management for improved HIV care in resource-limited settings. The healthcare system of any resource-limited system needs to ensure that leadership and governance at the health facilities need to be strengthened as they are critical in improving patient HIV care. Health facilities need to have adequate and competent service providers to run the HIV services to improve on the quality of care and patient experience with the services provided.

\section{Acknowledgement}

The following are acknowledged for their great contribution towards the success of this study; Prof: Everd Bikaitoha Maniple, Dr. Dyogo Nantamu, TAU team, Mildmay Research, and Ethics Committee, the Uganda Nation Council for Science and Technology, research assistants, health facility teams, and my family members.

[3] Wolf, Jason A., Niederhauser, V. Marshburn, Dianne and LaVela, Sherri L. (2014). "Defining Patient Experience," Patient Experience Journal: Vol. 1: Iss. 1, Article 3.

[4] Jackson, J. L., Chamberlin, J., \&Kroenke, K. (2001). Predictors of patient satisfaction. Social Science and Medicine, 52(4), 609-620. Doi:10.1016/S0277-9536(00)00164-7.

[5] Sophie Barton-Knott, (2017). The GENEVA/PARIS, 2017-UNAIDS new report. 
https://www.unaids.org/en/resources/presscentre/pre ssreleaseandstatementarchive/2017/july/20170720_P R_Global_AIDS_Update_2017.

[6] Musinguzi Joshua Ministry of Health, Uganda. (2019) Uganda Population-based HIV Impact Assessment (UPHIA) 2016-2017: Final Report. Kampala: Ministry of Health; July 2019. http://www.afro.who.int/sites/default/files/201708/UPHIA\%20Uganda\%20factsheet.pdf.

[7] Musinguzi Joshua. MoH (2012), Uganda AIDS Indicator Survey 2011. http://health.go.ug/docs/UAIS_2011_REPORT.pdf.

[8] Kambugu A.D (2016). Test and treat in Uganda: Perspectives from An Implementing/Academic Partner, Infectious Diseases Institute Makerere University College of Health Sciences. Southern African HIV clinicians' society, $3^{\text {rd }}$ biannual conference April 2016.

[9] Mobye K Anthony, and MoH (2016). Consolidated guidelines for HIV prevention, and treatment in Uganda 2016. https://differentiatedservicedelivery.org/portals/0/ad am/content/hvpzrp5yuusdpce $2 \mathrm{~m} 0 \mathrm{kmdq} /$ file/consolid ated $\% 20$ guidelines $\% 20$ for $\% 20$ prevention $\% 20$ and $\%$ 20treatment\%20of\%20hiv\%20in\%20uganda.pdf.

[10]UNFPA (2017). Uganda, the status of HIV prevention in 2017. Where are we in the fight against HIV. UNFPA Technical Division SRHB May 2017. https://uganda.unfpa.org/sites/default/files/pubpdf/Uganda_Status\%20of\%20HIV\%20Prevention.p df.

[11]Fonsah JY, Njamnshi AK, Kouanfack C, Qiu F, Njamnshi DM, Tagny CT, et al. (2017) Adherence to Antiretroviral Therapy (ART) in YaoundeÂCameroon: Association with Opportunistic Infections, Depression, ART Regimen and Side Effects. Plos One 12(1): e0170893. DOI: 10.1371/journal.pone.0170893.

[12] Bach Xuan Tran1*, Long Thanh Nguyen2, Nga Hoang Nguyen3, Quynh Van Hoang1 and Jongnam Hwang. Determinants of antiretroviral treatment adherence among HIV/AIDS patients: a multisite study.

[13]Bharatesh D. Basti, ${ }^{1}$ Venkatesha Mahesh, ${ }^{2}$ Dattatreya D. Bant, ${ }^{3}$ and Geeta V. Bathija (2017). Factors affecting antiretroviral treatment adherence among people living with human immunodeficiency virus/acquired immunodeficiency syndrome: A prospective study. Journal of family medicine and primary care.

[14] Oku O. Afiong, Eme T. Owoaje, Oboko O. Oku and Emmanuel Monjok (2014). Prevalence and Determinants of Adherence to Highly Active AntiRetroviral Therapy Amongst People Living with HIV/AIDS in a Rural Setting in South-South Nigeria. African Journal of Reproductive Health March 2014; 18(1): 134.

[15] Nyakato P, Kiragga AN, Kambugu A, et al. Correction of estimates of retention in care among a cohort of HIV-positive patients in Uganda in the period before starting ART: a sampling-based approach. BMJ Open 2018;8: e017487. doi:10.1136/ bmjopen-2017-017487.

[16] Mugisha Kenneth, Andrew Ocero, Edward Semafumu, Luigi Ciccio, Roland F. Muwanika, James Otim, Med Makumbi, (2009). Retention of HIV Positive Persons in Antiretroviral Therapy Programs in Post-Conflict Northern Uganda-Baseline Survey of 17 Health Units.

[17] Musomba R, Frank Mubiru, Shadia Nakalema, Hope Mackline, Ivan Kalule, Agnes N. Kiragga,1 Rosalind Parkes Ratanshi,1,2, and Barbara Castelnuovo. Describing Point of Entry into Care and Being Lost to Program in a Cohort of HIV Positive Pregnant Women in a Large Urban Centre in Uganda. Hindawi AIDS Research and Treatment, Volume 2017, Article ID 3527563, 6 pages https://doi.org/10.1155/2017/3527563.

[18]Elizabeth Horstmann, Jillian Brown, Fareesa Islam, Johanna Buck, and Bruce D. Agins, (2010). Retaining HIV-Infected Patients in Care: Where Are We? Where Do We Go from Here? Clinical Infectious Diseases 2010; 50:752-761.

[19] Park WB, Choe PG, Kim SH, Jo JH, Bang JH, Kim HB, Kim NJ, Oh M, Choe KW: One-year adherence to clinic visits after highly active antiretroviral therapy: a predictor of clinical progress in HIV patients. J Intern Med 2007, 261(3):268-275. [20] Smith E. O., J. D. Curb, and R. J. Hardy, "Clinic attendance in the hypertension detection and followup program," Hypertension, vol. 4, no. 5, pp. 710715,1982 . View at Google Scholar View at Scopus. 
[21]Nemes MI, Carvalho HB, Souza MF, (2004). Antiretroviral therapy adherence in Brazil. AIDS. https://www.ncbi.nlm.nih.gov/pubmed/15322479.

[22]Lucas GM, Chaisson RE, Moore RD, (1999). Highly active antiretroviral therapy in a large urban clinic: risk factors for virologic failure and adverse drug reactions. Ann Intern Med; 131(2):81-87.

[23] TenoRes Study Group (2016). Global epidemiology of drug resistance after failure of WHO recommended first-line regimens for adult HIV-1 infection: a multicentre retrospective cohort study. Lancet Infect Dis. 2016 May;16(5):565-575.

[24] Sethi A, Celentano D, Gange S, Moore R, Gallant J. Association between adherence to antiretroviral therapy and human immunodeficiency virus drug resistance. Clin Infect Dis 2003; 37(8):1112-1118.

[25] Gupta RK, Gregson J, Parkin N, Haile-Selassie

$\mathrm{H}$, Tanuri A, Andrade Forero L, Kaleebu P, Watera

C, Aghokeng A, Mutenda N, Dzangare J, Hone S, Hang ZZ, Garcia J, Garcia Z, Marchorro P, Beteta E, Giron A, Hamers R, Inzaule S, Frenkel LM, Chung MH, de Oliveira T, Pillay D, Naidoo K, Kharsany A, Kugathasan R, Cutino T, Hunt G, Avila Rios S, Doherty M, Jordan MR, Bertagnolio S. HIV-1 drug resistance before initiation or re-initiation of first-line antiretroviral therapy in low-income and middleincome countries: a systematic review and metaregression analysis. Lancet Infect Dis. 2018 Mar;18(3):346-355.

[26] Moore CG, Wilson-Witherspoon P, Probst JC, (2001). Time and money: effects of no-shows at a family practice residency clinic. Fam Med 2001; 33(7):522-527.

[27] Shumba c., L. Atuhaire, R. Imakit, R. Atukunda, and P. Memiah. Missed Doses and Missed Appointments: Adherence to ART among Adult Patients in Uganda. Hindawi Publishing Corporation, ISRN AIDS, Volume 2013, Article ID 270914, 7 pages, http://dx.doi.org/10.1155/2013/270914.

[28]Bigna Jean Joel R, Jean Jacques N Noubiap, Claudia S Plottel, Charles Kouanfack1, and Sinata Koulla-Shiro, (2014). Factors associated with nonadherence to scheduled medical follow-up appointments among Cameroonian children requiring
HIV care: a case-control analysis of the usual-care group in the MORE CARE trial.

[29] Setor Kunutsor,1 John Walley,1 Elly Katabira,2 Simon Muchuro,2 Hudson Balidawa,3 Elizabeth Namagala,3, and Eric Ikoona3, (2010). Clinic Attendance for Medication Refills and Medication Adherence amongst an Antiretroviral Treatment Cohort in Uganda: A Prospective Study. Hindawi Publishing Corporation AIDS Research and Treatment Volume 2010, Article ID 872396, 8 pages doi:10.1155/2010/872396.

[30]Uganda Virus Research Institute (2018). National HIV Drug Resistance Prevention, Monitoring and Surveillance Activities, National Status Report 2018.

[31]ECASA Group of Consultants LTD, (2018). Uganda AIDS Country Progress Report July 2017June 2018.

[32] Kolab Chhim1, Gitau Mburu2, Sovannary Tuot1, Ratana Sopha1, Vohith Khol3, Pheak Chhoun1 and Siyan (2018). Factors associated with viral non-suppression among adolescents living with HIV in Cambodia: a cross-sectional study. AIDS Research and Therapy. 15:20. https://doi.org/10.1186/s12981-018-0205-z.

[33] Katharina Kranzer, Stephen D. Lawn, Leigh F. Johnson, Linda-Gail Bekker, and Robin Wood, (2014). Community viral load and CD4 count distribution among people living with HIV in a South African township: implications for treatment as prevention. Europe PMC Funders Group. J Acquir Immune Defic Syndr. 2013 August 1; 63(4): 498-505. doi:10.1097/QAI.0b013e318293ae48.

[34] Bonner K, et al. Viral load monitoring as a tool to reinforce adherence: a systematic review. J Acquir Immune Defic Syndr. 2013;64(1):74-8.

[35] Nasuuna Esther, Joanita Kigozi1, Lillian Babirye, Alex Muganzi, Nelson K. Sewankambo and Damalie Nakanjako, (2018). Low HIV viral suppression rates following the intensive adherence counseling (IAC) program for children and adolescents with viral failure in public health facilities in Uganda. Nasuuna et al. BMC Public Health (2018) 18:1048. https://doi.org/10.1186/s12889-018-5964-x. 
[36] Martin Fischer and Gill Ereaut, (2012), Report: when doctors and patients talk: making sense of the consultation. The health foundation inspiring improvement.

[37] Institute of Medicine (1993). Committee on Monitoring Access to Personal Health Care Services. Access to health care in America. Washington, DC: National Academy Press; 1993.

[38] Agency for Healthcare Research and Quality (2011). National healthcare disparities report 2011.AHRQ Publication No. 12-0006 March 2012

[39] Sergei Savin, (n.d), Managing patient appointments in primary care. Graduate School of Business, 404 Uris Hall, Columbia University, New York, NY 10027.

[40]Lisa Brandenburg, Patricia Gabow, Glenn Steele, John Toussaint, and Bernard J. Tyson*(2015) Innovation and Best Practices in Health Care Scheduling. Institute of Medicine of the National Academies.

[41] Mallinson RK, Relf MV, Dekker D, Dolan K, Darcy A, Ford A, (2005). Maintaining normalcy: a grounded theory of engaging in $\mathrm{HIV}$-oriented primary medical care. ANS Adv Nurs Sci 2005; 28(3):265-277.

[42] Sendzik D. (2004) Retaining HIV-positive patients in care. In: Program and abstracts of the XV International AIDS Conference (Bangkok, Thailand). [43] Bradford J, Coleman S, Cunningham W. (2007). HIV System Navigation: an emerging model to improve HIV care access. AIDS Patient Care STDS. [44] Wohler-Torres BA, Valverde EE, AndersonMahoney P, Barragan M, Loughlin A, Metsch LR, (2002). System barriers to HIV medical care appointment compliance: results of the artas study. In: Program and abstracts of the XIV International AIDS Conference (Barcelona, Spain).

[45] Calsyn RJ, Klinkenberg WD, Morse GA, Miller J, Cruthis R, (2004). Recruitment, engagement, and retention of people living with HIV and cooccurring mental health and substance use disorders. AIDS Care.

[46] Niamat Ullah Ibne Hossain, Hunter Debusk, Md Mahmudul Hasan, Raed Jaradat, Mahmoud T. Khasawneh, (2017), Reducing Patient Waiting Time in an Outpatient Clinic: A Discrete Event Simulation
(DES) Based Approach, Abstract ID: 2220. Proceedings of the 2017 Industrial and Systems Engineering Conference K. Coperich, E. Cudney, H. Nembhard, eds.

[47]Emily Tse Lin Ho (2014), Improving waiting time and operational clinic flow in a tertiary diabetes center. BMJ Quality Improvement Reports 2014; u201918.w1006 doi: 10.1136/bmjquality. u201918.w1006.

[48]PHCPI (Primary Health Care Performance Initiative), (2018), improvement strategies model for access and timeliness. Version 1.0: Updated October 2018.

[49] NHS England, (n.d). Involving people in their own health and care: Statutory guidance for clinical commissioning groups and NHS England.

[50]Abdullah Khlofh Tabekhan1, Yahia Mater Alkhaldi, Abdullah Khalufah Alghamdi, (2018), Patient's satisfaction with consultation at primary health care centers in Abha City, Saudi Arabia. King Khalid University, Abha, Saudi Arabia.

[51] Kennedy, Denise M. MBA; Fasolino, John P. MD; and Gullen, David J. MD (2014) "Improving the patient experience through provider communication skills building,"Patient Experience Journal: Vol. 1: Iss. $1, \quad$ Article 10. Available at: http://pxjournal.org/journal/vol1/iss1/10.

[52]Agency for Healthcare Research and Quality (2019). More effort is needed to ensure pateints understand doctor's instructions. https://www.ahrq.gov data spotlight-health-literacy [53]Leonard L. Berry, Katie A. Deming, and Tracey S. Danaher, (2018). Improving Nonclinical and Clinical-Support Services: Lessons from Oncology. Mayo Foundation for Medical Education and Research. Published by Elsevier Inc.

[54] Shojania K, McDonald K, Wachter R, et al (n.d). Closing the quality gap: a critical analysis of quality improvement strategies_- Volume 7.

[55]PSIC (Professional Solutions Insurance Company), (2011), Why the Patient History Is So Important. issue 4 of 2011.

[56] Nick Summerton (2008). The medical history as a diagnostic technology. British Journal of General Practice. DOI: 10.3399/bjgp08X279779. 
[57] Institute of Medicine (2014. Facilitating patient understanding of discharge instructions: Workshop summary. Washington, DC: The National Academies Press.

[58] Tanya Bezreh, M Barton Laws, Tatiana Taubin,
Dena E Rifkin, and Ira B Wilson, (2011). Challenges to physician-patient communication about medication use: a window into the skeptical patient's world. Health Services Policy and Practice, University of California-USA. 